\title{
FINITE GROUPS WITH \\ REAL CONJUGACY CLASSES OF PRIME SIZE
}

by

\author{
Silvio Dolfi \\ Dipartimento di Matematica \\ Università di Firenze \\ 50134 Firenze, ITALY \\ E-mail: dolfi@math.unifi.it
}

\author{
Emanuele Pacifici \\ Dipartimento di Matematica \\ Università di Milano \\ 20133 Milano, ITALY \\ E-mail: pacifici@mat.unimi.it \\ and \\ Lucia Sanus \\ Departament d'Àlgebra \\ Facultat de Matemàtiques \\ Universitat de València \\ 46100 Burjassot, València, SPAIN \\ E-mail: lucia.sanus@uv.es
}


1. INTRODUCTION. It is known that the set $\operatorname{cs}(G)$ of sizes of the conjugacy classes of a finite group $G$ encodes nontrivial information about the structure of $G$. Many instances of the connection between arithmetical properties of $\operatorname{cs}(G)$ and structural properties of $G$ have been studied in the past two decades.

We consider a problem of similar nature concerning a smaller set of invariants, that is, the set $\mathrm{cs}_{\text {real }}(G)$ of sizes of the real conjugacy classes of $G$. Recall that a conjugacy class $g^{G}$ of $G$ is called real if $g^{G}=\left(g^{-1}\right)^{G}$, and the terminology is due to the following fact: these are precisely the conjugacy classes where every character of $G$ takes a real value. It has been shown that even this relatively small set has a remarkable connection with the structure of the group: see for instance $[\mathrm{CM}]$ and $[\mathrm{NST}]$.

We study here the structure of finite groups whose noncentral real conjugacy classes have all prime size.

THEOREM A. Let $G$ be a finite group. If all noncentral real conjugacy classes of $G$ have prime size, then $G$ is solvable. Furthermore, $\mathrm{cs}_{\text {real }}(G)$ is contained in a set of the kind $\{1,2\},\{1, p\}$ or $\{1,2, p\}$, where $p$ is an odd prime.

In fact, the claim concerning solvability in the above statement follows from the more general Theorem (3.1).

In the case when $\operatorname{cs}_{\text {real }}(G)=\{1,2, p\}$, we have a very neat description of the structure of $G$.

THEOREM B. Let $G$ be a finite group, and $p$ an odd prime. Then $\operatorname{cs}_{\text {real }}(G)=\{1,2, p\}$ if and only if $G=H \times O$, where $H$ is a dihedral group of order $2 p$ and $\operatorname{cs}_{\text {real }}(O)=\{1\}$.

The structure of finite groups whose real conjugacy classes are all central has been described in $[\mathrm{CM}]$; see Theorem (2.1) below.

We further give a description of the finite groups $G$ such that $\operatorname{cs}_{\text {real }}(G)=\{1,2\}$ (Theorem (3.5)) and $\mathrm{cs}_{\text {real }}(G)=\{1, p\}$ (Theorem (3.7)). We shall exploit the information provided by these results (together with Theorem B) in order to get the following general theorem.

THEOREM C. Let $G$ be a finite group. If all noncentral real conjugacy classes of $G$ have prime size, then $G$ has either a normal 2-complement or a normal Sylow 2-subgroup.

It may be worth recalling that several results concerning the sizes of the conjugacy classes of a finite group have a counterpart in the context of degrees of irreducible characters. The transposition to this context of the problem considered here is analyzed in [DPS], and Theorem A of this paper can be compared with Theorems A and B of that paper.

Throughout the following discussion, every abstract group is tacitly assumed to be finite. Also, "class" will stand for "conjugacy class".

2. PRELIMINARIES. Let $G$ be a group. An element $x$ of $G$ is called a real element if there exists a $g$ in $G$ such that $x^{g}=x^{-1}$. We denote by $\operatorname{Re}(G)$ the set of all real elements of $G$. 
A class $x^{G}=\left\{g^{-1} x g \mid g \in G\right\}$ is called real if $x$ is a real element of $G$ or, equivalently, if $x^{G}$ coincides with $\left(x^{-1}\right)^{G}$. As mentioned in the Introduction, we denote by

$$
\operatorname{cs}_{\text {real }}(G)=\left\{\left|x^{G}\right|=\left|G: \mathbf{C}_{G}(x)\right|: x \in \operatorname{Re}(G)\right\}
$$

the set of sizes of the real classes of $G$.

It is well known that the trivial class is the only real class of $G$ if and only if $G$ has odd order (see (iii) of Lemma (2.4)).

The groups whose real classes are all central are studied in [CM].

(2.1) THEOREM ([CM], Theorem 1.2). Let $G$ be a group. We have $\operatorname{cs}_{\text {real }}(G)=\{1\}$ if and only if $G=C \times D$, where $|C|$ is odd and $D$ is a 2-group with $\operatorname{Re}(D) \subseteq \mathbf{Z}(D)$.

The 2-groups $D$ such that $\operatorname{cs}_{\text {real }}(D)=\{1\}$ have been characterized in Proposition (4.7) of $[\mathrm{CM}]$ as the 2-groups satisfying the following condition: for $x, y \in D, x^{2}=y^{2}$ if and only if $\left(x y^{-1}\right)^{2}=1$.

The structure of the groups $G$ such that $\operatorname{cs}_{\text {real }}(G)$ consists just of odd numbers is also known.

(2.2) THEOREM ([DNT], Theorem 6.1). Let $G$ be a group. All real classes of $G$ have odd size if and only if $G$ has a normal Sylow 2-subgroup $D$ and $\operatorname{Re}(D) \subseteq \mathbf{Z}(D)$.

We shall also make use of the following result, which appears as Theorem $\mathrm{C}$ in [NST].

(2.3) THEOREM. Let $G$ be a group. All real classes of $G$ have 2-power size if and only if $G$ has a normal 2-complement $K$ and $\operatorname{Re}(G) \subseteq \mathbf{C}_{G}(K)$.

We next recall some known elementary facts about real elements and classes.

(2.4) LEMMA. Let $G$ be a group.

(i) If $x$ is a real element of $G$ and $\left|x^{G}\right|$ is odd, then $x^{2}=1$.

(ii) If $x$ is a real element of $G$, then every power of $x$ is a real element of $G$.

(iii) The identity is the unique real element of $G$ if and only if $|G|$ is odd.

(iv) If $N \unlhd G$ and $|G / N|$ is odd, then $\operatorname{Re}(G)=\operatorname{Re}(N)$.

Proof. (i) Let $x \in \operatorname{Re}(G)$. The inversion map yields a permutation on $x^{G}$. If $\left|x^{G}\right|$ is odd, then there must be an element $y$ in $x^{G}$ which is fixed by the inversion map. Therefore we get $y^{2}=1$, and clearly also $x^{2}=1$.

(ii) Let $x \in \operatorname{Re}(G)$, and let $g \in G$ be such that $x^{g}=x^{-1}$. Then $g$ acts as the inversion map on $\langle x\rangle$, hence every element in $\langle x\rangle$ is a real element of $G$.

(iii) If $|G|$ is even, then $G$ contains an involution, which is a nontrivial real element. Conversely, if $|G|$ is odd and $x$ is a real element of $G$ then, by (i), $x^{2}=1$ and hence $x=1$.

(iv) Let $x$ be in $\operatorname{Re}(G)$. Clearly $x N$ is in $\operatorname{Re}(G / N)$ and, since $n=|G / N|$ is odd, (iii) yields $x \in N$. Also, if $g \in G$ is such that $x^{g}=x^{-1}$, we get $x^{g^{n}}=x^{-1}$ and $g^{n} \in N$. This proves $\operatorname{Re}(G) \subseteq \operatorname{Re}(N)$. The converse inclusion is trivial.

(2.5) LEMMA. Let $G$ be a group, and let $x, y \in G$ be such that $x y=y x$. 
(i) Assume $G=\mathbf{C}_{G}(x) \mathbf{C}_{G}(y)$. Then $(x y)^{G}=x^{G} y^{G}$. If in addition $x$ and $y$ are in $\operatorname{Re}(G)$, then $x y \in \operatorname{Re}(G)$.

(ii) If $(o(x), o(y))=1$, or if $\langle x\rangle$ and $\langle y\rangle$ are normal in $G$ and have trivial intersection, then $\mathbf{C}_{G}(x y)=\mathbf{C}_{G}(x) \cap \mathbf{C}_{G}(y)$.

Proof. (i) Let $u$ and $v$ be in $G$. As $G=\mathbf{C}_{G}(x) \mathbf{C}_{G}(y)$, we can write $u v^{-1}=a^{-1} b$ for suitable $a \in \mathbf{C}_{G}(x)$ and $b \in \mathbf{C}_{G}(y)$. If now we set $g:=a u=b v$, we have

$$
x^{u} y^{v}=x^{a u} y^{b v}=(x y)^{g} .
$$

This proves $x^{G} y^{G} \subseteq(x y)^{G}$. The converse inclusion is trivial. If $x$ and $y$ are real in $G$, then $x^{-1} \in x^{G}$ and $y^{-1} \in y^{G}$, so $(x y)^{-1}=y^{-1} x^{-1}=x^{-1} y^{-1} \in x^{G} y^{G}=(x y)^{G}$ and hence $x y$ is real in $G$. Claim (ii) is straightforward.

Clearly, if $N$ is a subgroup of $G$, then $\operatorname{Re}(N) \subseteq \operatorname{Re}(G)$. Also, real elements of $G$ map to real elements of a factor group (under the natural homomorphism).

On the other hand, in general it is not true that a real element of a factor group has a real preimage under the natural homomorphism, but this holds for elements of odd order.

(2.6) LEMMA ([NST], Lemma 3.2). Let $N \unlhd G$, and suppose that $x N$ is a real element of odd order in $G / N$. Then there exists a real element $y$ of $G$ such that $y N=x N$.

One might compare the following proposition with (iii) of Lemma (2.4).

(2.7) PROPOSITION ([DNT], Proposition 6.4). The group $G$ has no nontrivial real elements of odd order if and only if $G$ has a normal Sylow 2-subgroup.

We finally need a result concerning coprime actions.

(2.8) PROPOSITION. Let $A$ be a group of odd order that acts faithfully on a nontrivial elementary abelian 2-group $V$ of order $2^{n}$. Assume that $\mathbf{C}_{V}(A)=1$. Then the following conditions are equivalent.

(i) For every nontrivial $x \in V,\left|A: \mathbf{C}_{A}(x)\right|$ is a prime number.

(ii) There exists a prime $p$ such that, for every nontrivial $x \in V,\left|A: \mathbf{C}_{A}(x)\right|=p$.

(iii) Either $|A|=p$, where $p$ is a prime, or $A$ is a nonabelian group of order $p q$, where $p, q$ are primes such that $q \mid(p-1, n)$ and

$$
p=\frac{2^{n}-1}{2^{n / q}-1} .
$$

Proof. It is trivial that (ii) implies (i).

From Lemma (4.3) of [DPS], it follows that (i) implies (iii). It hence remains to show that (iii) implies (ii). We may assume that $A$ is a nonabelian group of order $p q$, since if $|A|=p$ then (ii) is obvious. Note that $A$ is a Frobenius group with Frobenius kernel $P$ of order $p$, and $p$ is odd. By Maschke's Theorem, $V$ is a completely reducible $P$-module. Let $W$ be an irreducible $P$-submodule of $V$, with $|W|=2^{m}$. As $P$ acts faithfully on $V$, we can choose $W$ not to be a trivial $P$-module. Then, by Satz (II.3.10) of [H], $m$ is the smallest 
positive integer such that $p$ divides $2^{m}-1$. Now, from the relation (1), we see that $n \neq 6$. Hence, by Theorem (IX.8.3) of [HB], there exists a Zsigmondy prime divisor $s$ of $2^{n}-1$, that is, a prime $s$ such that $s$ divides $2^{n}-1$ but $s$ does not divide $2^{t}-1$ for all $1 \leq t<n$. Thus $s$ divides $\left(2^{n}-1\right) /\left(2^{n / q}-1\right)$, and therefore $s=p$. It follows that $m=n$, thus $V$ is an irreducible $P$-module and, in particular, we get $\mathbf{C}_{V}(P)=1$. By Theorem (15.16) of $[\mathrm{I}]$, it follows that $\left|\mathbf{C}_{V}(Q)\right|=2^{n / q}$ for every $Q \in \operatorname{Syl}_{q}(A)$. Observe now that, for distinct $Q_{1}, Q_{2} \in \operatorname{Syl}_{q}(A)$, we have $\left\langle Q_{1}, Q_{2}\right\rangle=A$ and hence $\mathbf{C}_{V}\left(Q_{1}\right) \cap \mathbf{C}_{V}\left(Q_{2}\right)=1$. By (1), we get that every nontrivial element $x \in V$ is centralized by a Sylow $q$-subgroup of $A$, and condition (ii) follows.

3. PROOFS. The part of Theorem A concerning solvability is implied by the following more general result.

(3.1) THEOREM. Let $G$ be a group. If all real class sizes of $G$ are either odd numbers or powers of 2 , then $G$ is solvable.

Proof. We argue by induction on the order of the group. Let $N$ be a maximal normal subgroup of $G$. Recalling that every real element $x$ of $N$ is a real element of $G$, and that $\left|x^{N}\right|$ divides $\left|x^{G}\right|$, we get that, for every $x \in \operatorname{Re}(N),\left|x^{N}\right|$ is either odd or a power of 2 . By induction, $N$ is solvable.

Assume that $G / N$ is a nonabelian simple group. By Feit-Thompson's Odd Order Theorem and Proposition (2.7), there exists a nontrivial $x N \in \operatorname{Re}(G / N)$ of odd order. Then, by (i) of Lemma (2.4), $\left|(x N)^{G / N}\right|$ is even. Now, by Lemma (2.6), there exists $y \in \operatorname{Re}(G)$ such that $y N=x N$ and, since $\left|(x N)^{G / N}\right|=\left|(y N)^{G / N}\right|$ divides $\left|y^{G}\right|$, it follows that $\left|(x N)^{G / N}\right|$ is power of a 2 . This contradicts a classical result by Burnside (see Theorem (3.9) of $[\mathrm{I}]$ ), and we conclude that $G / N$ must be solvable. The desired conclusion follows.

Observe that, in particular, Theorem (3.1) implies that every group whose real classes have all prime power size is solvable.

We next prove the second claim of Theorem A.

(3.2) THEOREM. Assume that every noncentral real class of a group $G$ has prime size. Then there exists a prime $p$ such that

$$
\operatorname{cS}_{\text {real }}(G) \subseteq\{1,2, p\}
$$

Proof. Assume first that $G$ has a (nontrivial) normal Sylow 2-subgroup $D$, and set

$$
U=\left\langle g \in \operatorname{Re}(G):\left|g^{G}\right| \text { is odd }\right\rangle
$$

Observe that, by (i) of Lemma (2.4), $U$ is an elementary abelian 2-group which is a subgroup of $\mathbf{Z}(D)$, whence we have $U \unlhd G$. Let $A=G / \mathbf{C}_{G}(U)$. Then $|A|$ is odd and, by Fitting's Lemma, $U=V \times \mathbf{C}_{U}(A)$, where $V=[A, U]$. Note that $A$ acts faithfully on $V$ and that $\mathbf{C}_{V}(A)=\mathbf{C}_{U}(A) \cap V=1$. Every nontrivial $x \in V$ is an involution, so $x \in \operatorname{Re}(G)$ and $\left|x^{G}\right|=\left|G: \mathbf{C}_{G}(x)\right|=\left|A: \mathbf{C}_{A}(x)\right|$ is a prime number. Then, by Proposition (2.8), 
there exists a prime $p$ such that $\left|A: \mathbf{C}_{A}(x)\right|=p$ for every nontrivial $x \in V$. Now, if $g \in \operatorname{Re}(G)$ and $\left|g^{G}\right|$ is odd, then $g=x y$ for suitable elements $x \in V$ and $y \in \mathbf{C}_{U}(A)$, and hence $\left|g^{G}\right|=\left|A: \mathbf{C}_{A}(x)\right| \in\{1, p\}$. It follows that $\operatorname{cs}_{\text {real }}(G) \subseteq\{1,2, p\}$.

We now assume that $G$ does not have a normal Sylow 2-subgroup. Thus, by Proposition (2.7), there exists a nontrivial element $x \in \operatorname{Re}(G)$ of odd order. Observe that, by (ii) of Lemma (2.4), we can assume $o(x)=p$, where $p$ is an odd prime. By (i) of Lemma (2.4), $\left|x^{G}\right|$ is even, and hence $\left|x^{G}\right|=\left|G: \mathbf{C}_{G}(x)\right|=2$. Let now $y$ be a nontrivial real element of $G$ such that $\left|y^{G}\right|=q$ is odd. Applying again (i) of Lemma (2.4), we see that $y$ is an involution.

If we assume $q \neq p$, then $\mathbf{C}_{G}(y)$ contains a Sylow $p$-subgroup of $G$, whence it contains $x$. As $\mathbf{C}_{G}(x)$ and $\mathbf{C}_{G}(y)$ have coprime index in $G$, by Lemma (2.5) we have $x y \in \operatorname{Re}(G)$ and

$$
\left|(x y)^{G}\right|=\left|G: \mathbf{C}_{G}(x) \cap \mathbf{C}_{G}(y)\right|=2 q,
$$

a contradiction. It follows again that $\operatorname{cs}_{\text {real }}(G) \subseteq\{1,2, p\}$.

(3.3) LEMMA. Let $G$ be a group, $x$ a nontrivial real element of $G$, and assume that $x$ has odd order. If the conjugacy class $x^{G}$ has prime size $q$, then $q=2$ and $\langle x\rangle \unlhd G$. Further, for every nontrivial $y \in\langle x\rangle$, we have $\mathbf{C}_{G}(y)=\mathbf{C}_{G}(x)$.

Proof. Since the order of $x$ is odd, by (i) of Lemma (2.4) the class of $x$ in $G$ has even size, and hence $q=2=\left|G: \mathbf{C}_{G}(x)\right|$. Also, as $x^{g}=x^{-1} \neq x$ for some $g \in G$, we get that $\mathbf{C}_{G}(x)$ is properly contained in $\mathbf{N}_{G}(\langle x\rangle)$, and hence $\langle x\rangle \unlhd G$. Finally, if $y$ is a nontrivial element of $\langle x\rangle$, then $y$ is real in $G$ by (ii) of Lemma (2.4). As $\mathbf{C}_{G}(x) \leq \mathbf{C}_{G}(y)$ and, since $y \neq y^{-1}$, we have $\mathbf{C}_{G}(y)<G$, it follows that $\mathbf{C}_{G}(y)=\mathbf{C}_{G}(x)$.

We are now going to prove Theorem B, which we state again.

(3.4) THEOREM. Let $G$ be a group, and $p$ an odd prime. Then $\operatorname{cs}_{\text {real }}(G)=\{1,2, p\}$ if and only if $G=H \times O$, where $H$ is a Dihedral group of order $2 p$ and $\operatorname{cs}_{\text {real }}(O)=\{1\}$.

Proof. Assume $\mathrm{cs}_{\text {real }}(G)=\{1,2, p\}$, and let $D$ be a Sylow 2-subgroup of $G$. If no nontrivial real element of $G$ has odd order, then $D \unlhd G$ by Proposition (2.7), and every real element of $G$ belongs to $D$ by (iv) of Lemma (2.4). Let $x, y \in \operatorname{Re}(G)$ be such that $\left|x^{G}\right|=\left|G: \mathbf{C}_{G}(x)\right|=p$ and $\left|y^{G}\right|=\left|G: \mathbf{C}_{G}(y)\right|=2$. Then $x \in \mathbf{Z}(D)$, and $G=$ $\mathbf{C}_{G}(x) \mathbf{C}_{G}(y)$. Therefore, by Lemma (2.5), $x y$ is a real element of $G$ and $x^{G} y^{G}=(x y)^{G}$. Thus, $\left|(x y)^{G}\right| \geq p>2$ implies $\left|(x y)^{G}\right|=p$. It follows that $x y \in \mathbf{Z}(D)$ and hence $y \in \mathbf{Z}(D)$, a contradiction.

By the discussion in the above paragraph, there exists a nontrivial real element $x$ of $G$ having odd order. Let $M=\mathbf{C}_{G}(x)$. By Lemma (3.3), for every $1 \neq y \in\langle x\rangle, \mathbf{C}_{G}(y)=M$ has index 2 in $G$. Let now $z$ be a real element of $G$ such that $\left|z^{G}\right|=p$. Observe that $z$ is an involution by (i) of Lemma (2.4). Now, if any $1 \neq y \in\langle x\rangle$ centralizes $z$ then, by Lemma (2.5), $y z \in \operatorname{Re}(G)$ and $\left|(y z)^{G}\right|=2 p$, a contradiction. Therefore, $\mathbf{C}_{G}(z)$ intersects $\langle x\rangle$ trivially. In particular, $z \notin M$ and hence $\langle z\rangle$ is a complement of $M$ in $G$.

Furthermore, as $\langle x\rangle \cap \mathbf{C}_{M}(z)=1$ and $\left|M: \mathbf{C}_{M}(z)\right|$ divides $\left|G: \mathbf{C}_{G}(z)\right|=p$, it follows that $M=\langle x\rangle \times \mathbf{C}_{M}(z)$ and that $|\langle x\rangle|=p$. Write $H=\langle x, z\rangle$ and $O=\mathbf{C}_{M}(z)$. Then $G=H \times O$ and, since $z$ acts as the inversion map on $\langle x\rangle$ (recall that, by Lemma (3.3), $\langle x\rangle$ 
is normal in $G), H$ is a dihedral group of order $2 p$. Finally, as cs cseal $(H \times O)=\{n m \mid n \in$ $\left.\operatorname{cs}_{\text {real }}(H), m \in \mathrm{cs}_{\text {real }}(O)\right\}$, and $\operatorname{cs}_{\text {real }}(H)=\{1,2, p\}$, it follows that $\operatorname{cs}_{\text {real }}(O)=\{1\}$.

Conversely, it is clear that if $H$ is a dihedral group of order $2 p$ and $\operatorname{cs}_{\text {real }}(O)=\{1\}$, then $\mathrm{cs}_{\text {real }}(H \times O)=\operatorname{cs}_{\text {real }}(H)=\{1,2, p\}$.

We move now toward the proof of Theorem C. We shall take advantage of Theorem (3.5) and Theorem (3.7), which provide structural information concerning groups whose noncentral real classes have all the same (prime) size.

(3.5) THEOREM. Let $G$ be a finite group. Then $\operatorname{cs}_{\text {real }}(G)=\{1,2\}$ if and only if $G=H \times O$, where $\mathrm{cs}_{\text {real }}(O)=\{1\}$, and either

(a) $H$ is a 2-group with $\mathrm{cs}_{\text {real }}(H)=\{1,2\}$; or

(b) $H=A D$, where $A$ is a normal abelian 2-complement of $H$ and $D$ is a Sylow 2-subgroup of $H, C=\mathbf{C}_{D}(A)$ has index 2 in $D$, and $\operatorname{Re}(D) \subseteq \mathbf{Z}(C)$.

Proof. Assume $\mathrm{cs}_{\mathrm{real}}(G)=\{1,2\}$. Then, by Theorem (2.3), $G$ has a normal 2-complement $K$ and $\operatorname{Re}(G) \subseteq \mathbf{C}_{G}(K)$. Let $D$ be a Sylow 2-subgroup of $G$.

If $G$ has no nontrivial real element of odd order then, by Proposition (2.7), we get $G=D \times K$, and therefore (a) holds.

We may hence assume that $K \cap \operatorname{Re}(G) \neq\{1\}$, so that the normal subgroup $A=$ $\langle K \cap \operatorname{Re}(G)\rangle$ of $G$ is nontrivial. We claim that $B=\mathbf{C}_{G}(A)$ has index 2 in $G$. Let $x_{1}, x_{2} \in K \cap \operatorname{Re}(G)$ and $B_{i}=\mathbf{C}_{G}\left(x_{i}\right), i=1,2$. If $B_{1} \neq B_{2}$ then, by Lemma (3.3), $\left\langle x_{1}\right\rangle \cap\left\langle x_{2}\right\rangle=1$ and $\left\langle x_{1}\right\rangle,\left\langle x_{2}\right\rangle \unlhd G$. As $B_{1} B_{2}=G$, Lemma (2.5) implies that $x_{1} x_{2} \in \operatorname{Re}(G)$ and that $\left|\left(x_{1} x_{2}\right)^{G}\right|=\left|G: B_{1} \cap B_{2}\right|>2$, a contradiction.

Hence $B$ has index 2 in $G$. In particular, $A \leq \mathbf{Z}(K)$ and $C=\mathbf{C}_{D}(A)$ is a subgroup of index 2 in $D$. Note that $D / C$ inverts each element of $K \cap \operatorname{Re}(G)$ and hence it inverts each element of $A$, so $\mathbf{C}_{A}(D)=1$.

Recall that if $x A \in \operatorname{Re}(G / A)$ has odd order then, by Lemma (2.6), $x A=y A$ for some $y \in \operatorname{Re}(G)$. As $x A \in K / A$, then $y \in \operatorname{Re}(G) \cap K \subseteq A$ and hence $x A$ is the identity element of $G / A$. By Proposition (2.7) it follows that $D A / A$ is a normal subgroup of $G / A$ and hence $[K, D] \leq A$. Then, by coprimality, $K=A \mathbf{C}_{K}(D)$. As $A \cap \mathbf{C}_{K}(D)=\mathbf{C}_{A}(D)=1$ and $A \leq \mathbf{Z}(K)$, we have $K=A \times \mathbf{C}_{K}(D)$ and hence $G=H \times O$, where $H=A D$ and $O=\mathbf{C}_{K}(D)$. As $|O|$ is odd, by (iii) of Lemma $(2.4), \operatorname{cs}_{\text {real }}(O)=\{1\}$.

Let now $u \in \operatorname{Re}(D)$. We have $u \in \operatorname{Re}(G)$, so that $A \leq \mathbf{C}_{G}(u)$ and $u$ lies in $C$. If $u$ is in $\mathbf{Z}(D)$, then $u$ is indeed in $\mathbf{Z}(G)$. We can therefore assume $u \notin \mathbf{Z}(D)$, so that $\left|G: \mathbf{C}_{G}(u)\right|=\left|D: \mathbf{C}_{D}(u)\right|=2$. If $\mathbf{C}_{D}(u) \neq C$ then, taking a nontrivial $x \in \operatorname{Re}(G) \cap A$, we get $\mathbf{C}_{G}(x) \mathbf{C}_{G}(u)=G$. By Lemma (2.5), $x u$ is real in $G$ and $\left|(x u)^{G}\right|=\left|G: \mathbf{C}_{G}(x u)\right|=$ $\left|G: \mathbf{C}_{G}(x) \cap \mathbf{C}_{G}(u)\right|=4$, a contradiction. Therefore $\mathbf{C}_{D}(u)=C$, whence $u \in \mathbf{Z}(C)$, as desired.

We now show, conversely, that from either (a) or (b) it follows that $\operatorname{cs}_{\text {real }}(G)=\{1,2\}$. Since $\operatorname{cs}_{\text {real }}(H \times O)=\mathrm{cs}_{\text {real }}(H)$, we may assume that (b) holds and show that $\operatorname{cs}_{\text {real }}(H)=$ $\{1,2\}$. Let $g \in \operatorname{Re}(H)$; we can write $g=x y$, where $x, y \in\langle g\rangle, o(x)$ is odd and $o(y)$ is a power of 2. By (ii) of Lemma (2.4) we see that $y \in \operatorname{Re}(H)$, whence there exists $z \in H$ such that $y^{z}=y^{-1}$. By taking a suitable power of $z$, we may assume that $z$ is a 2-element. So, $Y=\langle y, z\rangle$ is a 2-group and, up to conjugation in $H$, we may assume that $Y \leq D$. Thus, $y \in \operatorname{Re}(D) \subseteq \mathbf{Z}(C)$. Now, since $x \in A$ centralizes $A C$, we get 
$\mathbf{C}_{H}(g)=\mathbf{C}_{H}(x) \cap \mathbf{C}_{H}(y) \geq A C$ and hence $\left|g^{H}\right| \leq 2$. On the other hand, by Proposition (2.7) there is an element of odd order $g \in \operatorname{Re}(H)$, and by (i) of Lemma (2.4) we have $\left|g^{H}\right|=2$. Thus, $\operatorname{cs}_{\text {real }}(G)=\operatorname{cs}_{\text {real }}(H)=\{1,2\}$.

As usual, given a prime $p$ and a $p$-group $P$, we denote by $\Omega(P)$ the subgroup generated by the elements of order $p$ in $P$.

(3.6) LEMMA. Let $A$ be a group of odd order that acts faithfully on a 2-group $D$. Assume that $\operatorname{Re}(D) \subseteq \mathbf{Z}(D)$, and write $\Omega:=\mathbf{\Omega}(\mathbf{Z}(D))$. Then $\Omega=\operatorname{Re}(D), A$ acts faithfully on $V=[\Omega, A]$, and $\mathbf{C}_{V}(A)=1$.

Proof. Since $\operatorname{Re}(D) \subseteq \mathbf{Z}(D)$, the nontrivial real elements of $D$ coincide with the central involutions of $D$, that is, $\Omega=\operatorname{Re}(D)$. By Fitting's Lemma, $\Omega=V \times \mathbf{C}_{\Omega}(A)$, and hence $\mathbf{C}_{V}(A)=1$.

Let now $B=\mathbf{C}_{A}(V)$ and observe that $B$ fixes all the real elements of $D$. By Lemma (3.3) of [NST], then $B$ fixes all the real valued irreducible characters of $D$ and, in particular, $B$ fixes all the irreducible characters of the factor group $D / \Phi(D)$. Hence, by Brauer's Permutation Lemma (6.32) of $[\mathrm{I}], B$ acts trivially on $D / \boldsymbol{\Phi}(D)$. By coprimality, it follows that $B \leq \mathbf{C}_{A}(D)=1$, whence $A$ acts faithfully on $V$.

(3.7) THEOREM. Let $G$ be a finite group, $p$ an odd prime and $D$ a Sylow 2-subgroup of $G$. Then csreal $_{\text {rea }}(G)=\{1, p\}$ if and only if $D \unlhd G, \operatorname{Re}(D) \subseteq \mathbf{Z}(D)$ and, denoting by $K$ a 2-complement of $G$, either

(a) $\left|K / \mathbf{C}_{K}(D)\right|=p$; or

(b) $K / \mathbf{C}_{K}(D)$ is a nonabelian group of order $p q$, where $q$ is a prime. If $n$ is such that $2^{n}=|[\boldsymbol{\Omega}(\mathbf{Z}(D)), K]|$, we have $q \mid(p-1, n)$ and $p=\left(2^{n}-1\right) /\left(2^{n / q}-1\right)$.

Proof. If $\operatorname{cs}_{\text {real }}(G)=\{1, p\}$ then, by Theorem $(2.2), D$ is normal in $G$ and $\operatorname{Re}(D) \subseteq \mathbf{Z}(D)$.

Let $K$ be a 2-complement of $G$; set $A=K / \mathbf{C}_{K}(D)$ and $\Omega=\Omega(\mathbf{Z}(D))$. By Lemma (3.6), $\Omega=\operatorname{Re}(D), A$ acts faithfully on $V=[\Omega, A]$ and $\mathbf{C}_{V}(A)=1$. Now, if $x$ is a nontrivial element of $V$, then $\left|A: \mathbf{C}_{A}(x)\right|=\left|G: \mathbf{C}_{G}(x)\right| \in \operatorname{cs}_{\text {real }}(G)$ and hence $\left|A: \mathbf{C}_{A}(x)\right|=p$ for every nontrivial $x \in V$, since $\mathbf{C}_{V}(A)=1$. We can hence apply Proposition (2.8) and get that either (a) or (b) is satisfied.

Assume, conversely, that $G$ has a normal Sylow 2-subgroup $D$, with $\operatorname{Re}(D) \subseteq \mathbf{Z}(D)$. Assume also that, if $K$ is a 2-complement of $G$ and $A=K / \mathbf{C}_{K}(D)$, either (a) or (b) is satisfied. Let $\Omega=\boldsymbol{\Omega}(\mathbf{Z}(D))$. By Lemma (3.6) we know that $\Omega=\operatorname{Re}(D)$, that $A$ acts faithfully on $V=[\Omega, A]=[\Omega, K]$ and that $\mathbf{C}_{V}(A)=1$. Then, applying Proposition (2.8), it follows that $\left|A: \mathbf{C}_{A}(x)\right|=p$ for every nontrivial $x \in V$. Let now $g$ be a real element of $G$. By (iv) of Lemma (2.4) and Fitting's Lemma, $g \in \operatorname{Re}(D)=\Omega=V \times \mathbf{C}_{\Omega}(K)$. Write $g=x y$ with $x \in V$ and $y \in \mathbf{C}_{\Omega}(K)$. Since $\mathbf{C}_{\Omega}(K) \leq \mathbf{Z}(G)$, we have $\mathbf{C}_{G}(g)=\mathbf{C}_{G}(x)$, and hence $\left|g^{G}\right|=\left|G: \mathbf{C}_{G}(x)\right|=\left|A: \mathbf{C}_{A}(x)\right|$. Since $V \neq 1$, we get $\operatorname{cs}_{\text {real }}(G)=\{1, p\}$.

Now, Theorem $\mathrm{C}$ follows at once. 
Proof of Theorem C. Assume that all noncentral real classes of $G$ have prime size. Then by Theorem (3.2), we have $\operatorname{cs}_{\text {real }}(G) \subseteq\{1,2, p\}$ for some odd prime $p$. By Theorems (2.1), (3.4) and (3.5), if either $\operatorname{cs}_{\text {real }}(G) \subseteq\{1,2\}$ or $\operatorname{cs}_{\text {real }}(G)=\{1,2, p\}$, then $G$ has a normal 2-complement. If $\operatorname{cs}_{\text {real }}(G)=\{1, p\}$, then by Theorem (3.7) $G$ has a normal Sylow 2 -subgroup.

\section{REFERENCES}

[DNT] S. Dolfi, G. Navarro, P. Tiep, Primes dividing the degrees of the real characters, Math. Z. 259 (2008), $755-774$.

[DPS] S. Dolfi, E. Pacifici, L. Sanus, Finite groups with real valued irreducible characters of prime degree, J. Algebra, in press.

[CM] D. Chillag, A. Mann, Nearly odd-order and nearly real finite groups, Comm. Algebra 26 (1998), 2041-2064.

[H] B. Huppert, Endliche Gruppen I, Springer, Berlin, 1967.

[HB] B. Huppert, N. Blackburn, Finite groups II, Springer, Berlin, 1982.

[I] I. M. Isaacs, Character Theory of Finite Groups, Dover, New York, 1994.

[NST] G. Navarro, L. Sanus, P.H. Tiep, Real characters and degrees, Israel J. Math., in press. 